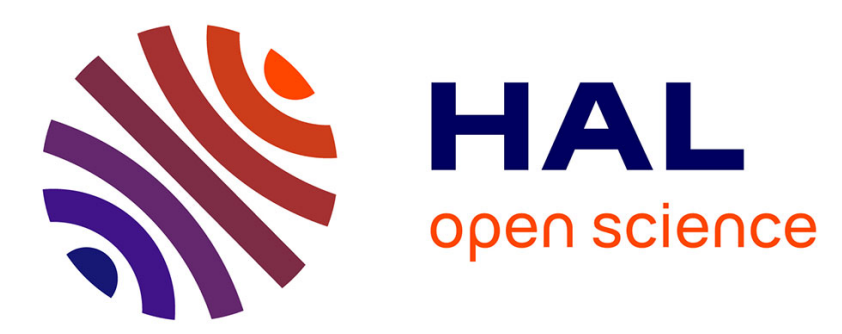

\title{
Dynamic Attitude Planning for Trajectory Tracking in Thrust-Vectoring UAVs
}

Davide Invernizzi, Marco Lovera, Luca Zaccarian

\section{To cite this version:}

Davide Invernizzi, Marco Lovera, Luca Zaccarian. Dynamic Attitude Planning for Trajectory Tracking in Thrust-Vectoring UAVs. IEEE Transactions on Automatic Control, 2020, 65 (1), pp.453-460. 10.1109/TAC.2019.2919660 . hal-03029040

\section{HAL Id: hal-03029040 https://hal.science/hal-03029040}

Submitted on 27 Nov 2020

HAL is a multi-disciplinary open access archive for the deposit and dissemination of scientific research documents, whether they are published or not. The documents may come from teaching and research institutions in France or abroad, or from public or private research centers.
L'archive ouverte pluridisciplinaire HAL, est destinée au dépôt et à la diffusion de documents scientifiques de niveau recherche, publiés ou non, émanant des établissements d'enseignement et de recherche français ou étrangers, des laboratoires publics ou privés. 


\title{
Dynamic attitude planning for trajectory tracking in thrust-vectoring UAVs
}

\author{
Davide Invernizzi, Marco Lovera and Luca Zaccarian
}

\begin{abstract}
This paper addresses the trajectory tracking control problem for underactuated UAVs, with specific focus on vehicles with thrustvectoring capabilities. According to the different actuation mechanisms, the most common UAV platforms can achieve only a partial decoupling of attitude and position tasks. Since position tracking is of utmost importance for applications involving aerial vehicles, we propose a control scheme in which position tracking is the primary objective. To this end, this work exploits the concept of attitude planner, a dynamical system through which the desired attitude reference is processed to guarantee the satisfaction of the primary objective: the attitude tracking task is considered as a secondary objective which can be realized as long as the desired trajectory satisfies specific trackability conditions.
\end{abstract}

Index Terms-UAVs, thrust vectoring, trajectory tracking

\section{INTRODUCTION}

The increasing demand of complex and challenging applications involving Vertical-Take-Off and Landing Unmanned Aerial Vehicles (VTOL UAVs) has led to the design of novel configurations to overcome the maneuverability limitation of standard platforms. In particular, multirotor UAVs with coplanar propellers cannot fulfill at the same time attitude and position tasks. We refer to these platforms as vectored-thrust UAVs because their propulsive system can deliver a control force only along a fixed direction within the airframe.

By designing an actuation mechanism that can change the thrust direction within the airframe, more complex maneuvering can be performed. Such platforms, which we call thrust-vectoring UAVs, can be realized by mounting the propellers in a non-coplanar fashion [3], [5] or by employing servo-actuators to dynamically adjust their orientation [8], [9], [19]. When the thrust vectoring capabilities are limited, the tracking control problem is challenging since only a partial decoupling is possible between the attitude and position objectives. In particular, our work starts by showing that it is impossible to track an arbitrary full pose trajectory (independent attitude and position) also for thrust-vectoring configurations, when the vectoring capability is limited. In this case a viable approach is to prioritize control objectives, for instance by subduing the realization of attitude tracking to the achievement of position tracking [5], [8]. While much literature is devoted to the trajectory tracking problem for the vectored-thrust configuration [7], [11], [13], [17], [18], [21], few works address the thrust-vectoring one [5], [8], [12].

The control design that we propose is developed in two stages. The first step is the design of a control force and torque that ensure robust tracking of any desired trajectory that possesses certain smoothness and boundedness properties. In the second step, we design a dynamic attitude planner to account for the system underactuation and to enforce prioritization of the position tracking objective over the attitude one in the spirit of ideas proposed in [5], [8]. In developing our control law we take as well inspiration from [18], in which the emphasis was on defining classes of position and attitude controllers that stabilize the UAV at a given position. In the same way, our control design parametrizes a family of control solutions to the tracking problem for underactuated UAVs provided that they possess some basic properties. Moreover, our contribution extends the results of [18], which only deals with stabilization (constant reference) and vectored-thrust platforms and the results of

D. Invernizzi, M. Lovera are with Department of Aerospace Science and Technology, Politecnico di Milano, Via La Masa 34, 20156, Milano, Italy.

L. Zaccarian is with LAAS-CNRS, Université de Toulouse, CNRS, Toulouse, France and with Dipartimento di Ingegneria Industriale, University of Trento, Italy zaccarian@laas.fr
[11], which only deals with a static attitude planning and vectoredthrust platforms. This extension is non-trivially accomplished here by characterizing a non-autonomous closed-loop by way of setvalued maps and it allows considering a large class of attitude control laws that do not necessarily make the attitude error subsystem autonomous nor exponentially stable [5], [8]. As in our previous work [11], the control design leaves freedom also in the choice of the position feedback stabilizer, provided that it guarantees certain properties: a basic requirement is the position dynamics be Input-toState Stable (ISS) with respect to small inputs. Such property is ensured by employing, e.g., nested saturations-based stabilizers [17]. Then, we show that our control design provides robust tracking with semi-global properties. To achieve this result, we study the stability of the closed-loop interconnection between the attitude and position error systems within the framework of differential inclusions. This approach simplifies the analysis of the corresponding cascaded non-autonomous system: the proof technique relies on casting the control problem as a stability problem for a compact attractor with dynamics satisfying regularity conditions, which ensures robustness of the stability property against a very large class of (sufficiently small) perturbations [6, Chapter 7]. Our proof is based on reduction theorems [14] which have been exploited with a different control strategy in [16] to address set-point tracking (stabilization). Finally, we show that existing attitude planning strategies developed for vectored-thrust [13] and thrust-vectoring platforms [12] fit within the proposed robust control design framework.

Notation. The canonical basis in $\mathbb{R}^{n}$ is denoted as $e_{i}:=$ $(0, \ldots, 1, \ldots, 0)$ for $i \in\{1, \ldots, n\}$ and the identity matrix in $\mathbb{R}^{n \times n}$ is denoted as $I_{n}:=\left[e_{1} \cdots e_{i} \cdots e_{n}\right]$. Given $x=$ $\left(x_{1}, \ldots, x_{n}\right) \in \mathbb{R}^{n},\|x\|:=\sqrt{x_{1}^{2}+\ldots+x_{n}^{2}}$ is the Euclidean norm while for a matrix $A \in \mathbb{R}^{n \times n},\|A\|_{F}:=\sqrt{\operatorname{tr}\left(A^{T} A\right)}$ is the Frobenius norm and $\operatorname{skew}(A):=\frac{A-A^{T}}{2}$ is the skew-symmetric part of $A . A \in \mathbb{R}_{>0}^{n \times n}\left(\mathbb{R}_{<0}^{n \times n}\right)$ denotes a positive (negative) definite matrix. The minimum and maximum eigenvalues of $A \in \mathbb{R}^{n \times n}$ are $\lambda_{m}(A)$ and $\lambda_{M}(A)$, respectively, and $\operatorname{skew}(A):=\frac{A-A^{T}}{2}$ is the skew-symmetric part of $A$. Set $\mathrm{SO}(3):=\left\{R \in \mathbb{R}^{3 \times 3}{ }^{2}: R^{T} R=\right.$ $\left.I_{3}, \operatorname{det}(R)=1\right\}$ denotes the third-order Special Orthogonal group. The normalized distance of $R \in \mathrm{SO}(3)$ with respect to $I_{3}$, induced by the Frobenius norm, is denoted as $\|R\|_{\mathrm{SO}(3)}:=\frac{1}{8} \| R-$ $I_{3} \|_{F}=\sqrt{\frac{1}{4} \operatorname{tr}\left(I_{3}-R\right)} \in[0,1]$. Given $\omega \in \mathbb{R}^{3}$, the hat map $\hat{\imath}: \mathbb{R}^{3} \rightarrow \mathfrak{s o}(3):=\left\{\Omega \in \mathbb{R}^{3 \times 3}: \Omega=-\Omega^{T}\right\}$ is such that $\hat{\omega} y=\omega \times y, \forall y \in \mathbb{R}^{3}$ and $\times$ represents the cross product in $\mathbb{R}^{3}$. The inverse of the hat map is the vee map, denoted as $(\cdot)^{\vee}: \mathfrak{s o}(3) \rightarrow \mathbb{R}^{3}$. The set of piecewise-continuous and bounded functions is denoted as $L_{\infty}$. We use standard comparison functions: a function $\alpha: \mathbb{R}_{\geq 0} \rightarrow \mathbb{R}_{\geq 0}$ is of class $K$ if it is zero at zero, strictly increasing, and continuous. It is of class $K_{\infty}$ if it is also unbounded. A function $\beta: \mathbb{R}_{\geq 0} \times \mathbb{R}_{\geq 0} \rightarrow \mathbb{R}_{\geq 0}$ is of class $K L$ if it is of class $K$ in the first argument and nonincreasing and converging to zero as its second argument tends to $+\infty$.

\section{DYNAMICAL MODELS FOR THRUST-VECTORING UAVS}

This section recalls the simplified dynamical model of multirotor UAVs that is usually employed for control design purposes. In order to obtain a control design independent from the specific actuation mechanism, we will assume the control wrench $w_{c}:=$ $\left(f_{c}, \tau_{c}\right)$, where $f_{c} \in \mathbb{R}_{3}$ and $\tau_{c} \in \mathbb{R}_{3}$ are the control force and torque, respectively, to be the control variable. Then, we will resort to approximate models of the wrench map (from physical 
inputs to delivered wrench), according to most common actuation mechanisms. The equations of motion for control design are:

$$
\begin{aligned}
\dot{x} & =v \\
\dot{R} & =R \hat{\omega} \\
m \dot{v} & =-m g e_{3}+R f_{c} \\
J \dot{\omega} & =-\hat{\omega} J \omega+\tau_{c},
\end{aligned}
$$

where $x \in \mathbb{R}^{3}$ and $v \in \mathbb{R}^{3}$ are position and velocity of the center of mass in the inertial frame, respectively, $R=\left[\begin{array}{lll}b_{1} & b_{2} & b_{3}\end{array}\right] \in \mathrm{SO}(3)$ is the rotation matrix describing the attitude of the body fixed frame $\mathcal{F}_{B}:=\left\{b_{1}, b_{2}, b_{3}\right\}$ with respect to the inertial frame, $\omega \in \mathbb{R}^{3}$ is the body angular velocity, $m \in \mathbb{R}_{>0}$ and $J=J^{T} \in \mathbb{R}_{>0}^{3 \times 3}$ are the UAV mass and inertia matrix with respect to the principal axes.

When the range of the map from the set of physical inputs $U$ to the delivered wrench, namely $U \ni u \mapsto w_{c}(u)$, spans $\mathbb{R}^{6}$, the system is fully actuated. In the following, the control torque $\tau_{c}$ is assumed to span $\mathbb{R}^{3}$, whilst the actuation mechanism allows to deliver the control force $f_{c}$ only on a compact subset of $\mathbb{R}^{3}$. Within this category of UAVs, a further classification distinguishes between vectored-thrust [11] and thrust-vectoring configurations [5]. As per the first class, the control force can be delivered only in the common direction of the thrust axes of the propellers:

$$
f_{c_{3}}(t)>0, \quad f_{c_{1}}(t)=f_{c_{2}}(t)=0 \quad \forall t \geq 0 .
$$

This configuration is adopted in most multirotor UAVs thanks to the inherent mechanical simplicity combined with good performance in many flight conditions. The second class comprises UAVs with thrust-vectoring capabilities such as multirotor UAVs both with fixed-tilted [5] or dynamically tiltable propellers [19]. While the dynamically tiltable configuration makes the system fully actuated if the tilt angle of the servo-actuators is not limited, this is not true for the fixed-tilted configuration, for which the maximum inclination at which the rotors are mounted is limited by the efficiency loss: the power consumption in hover is large and increases with the inclination of the propellers. For the latter configuration, the control force $f_{c}$ has to satisfy:

$$
0<\cos \left(\theta_{M}\right) \leq \frac{f_{c}(t)^{T} e_{3}}{\left\|f_{c}(t)\right\|}=\cos \left(\theta_{c}(t)\right) \quad \forall t \geq 0,
$$

where $\theta_{M}$ is the maximum tilt angle and $\|\cdot\|$ represents the Euclidean norm . Finally, for all the cases above, we assume that the control force is bounded by a positive scalar $T_{M} \in \mathbb{R}_{>0}$, namely $\left\|f_{c}(t)\right\| \leq T_{M} \forall t \geq 0$. This constraint is required to account for actuators saturations, in particular the limited spinning velocity of propellers.

\section{CONTROL PROBLEM STATEMENT}

This work deals with the tracking control problem for the system described by (1)-(4). The objective is to track a reference $t \mapsto\left(R_{d}(t), \omega_{d}(t), x_{d}(t), v_{d}(t)\right) \in T \mathrm{SO}(3) \times \mathbb{R}^{3} \times \mathbb{R}^{3}$, where $T \mathrm{SO}(3):=\mathrm{SO}(3) \times \mathbb{R}^{3}$ is the trivial tangent bundle of $\mathrm{SO}(3)$.

When considering the actuation limitations shown in the previous section, the tracking of an arbitrary reference trajectory in $T \mathrm{SO}(3) \times \mathbb{R}^{3} \times \mathbb{R}^{3}$ is not possible due to the platform underactuation. However, platforms with thrust-vectoring capabilities can achieve a certain degree of decoupling between attitude and position tracking when the corresponding trajectories are compatible in some sense. To suitably represent and exploit these degrees of freedom, let us first compute the steady state (short, ss) control wrench, which is obtained by inverting the system dynamics:

$$
\begin{aligned}
& f_{c}^{s s}(t):=m R_{d}^{T}(t)\left(\dot{v}_{d}(t)+g e_{3}\right) \\
& \tau_{c}^{s s}(t):=J \dot{\omega}_{d}(t)+\hat{\omega}_{d}(t) J \omega_{d}(t) .
\end{aligned}
$$

For arbitrary position and attitude trajectories, it is likely that $f_{c}^{s s}$ will not be compatible with the actuation limitations (5) and (6). On the other hand, because position tracking is mandatory in aerial

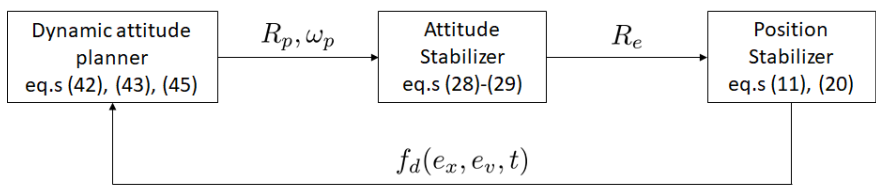

Fig. 1. Control scheme: cascade interconnection.

applications, equation (7) suggests that the desired attitude can be properly modified to be compliant with the actuation constraints. Indeed, according to equation (7), the control force is obtained by rotating the vector $m\left(\dot{v}_{d}+g e_{3}\right)$ by $R_{d}^{T}$. The rationale behind the proposed control is to prioritize position over orientation tracking, as already suggested by [8] and [5]. We will propose a strategy to track the desired attitude as long as it allows to follow the desired position. Whenever this condition cannot be granted, only the closest feasible attitude will be tracked. We will exploit the fact that when the control force is delivered along the vertical direction of the body frame $\left(b_{3}\right)$ (vectored-thrust case), the constraint (6) for the thrust-vectoring case is satisfied as well.

Before going on, the following assumptions (not too restrictive for standard applications) are required to hold for the desired trajectory.

Assumption 1: Smoothness and boundedness of the desired trajectory. The desired trajectory $t \mapsto\left(R_{d}(t), \omega_{d}(t), x_{d}(t), v_{d}(t)\right) \in$ $T S O(3) \times \mathbb{R}^{3} \times \mathbb{R}^{3}$ satisfies $\dot{R}_{d}(t)=R_{d}(t) \hat{\omega}_{d}(t)$ and $\dot{x}_{d}(t)=$ $v_{d}(t)$, for all $t \geq 0$, and is continuously differentiable up to the second order. Furthermore, the desired linear acceleration $\dot{v}_{d}(\cdot)$ and angular velocity $\omega_{d}(\cdot)$ are bounded.

\section{ROBUST STABILIZATION OF THE ERROR DYNAMICS}

In this section we show the design of a control law that ensures position tracking for any bounded and sufficiently smooth trajectory with no restriction on the initial position error and some restriction on the initial attitude error. Specifically, we will show that the system dynamics can be represented by a feedback interconnection in which an attitude planner provides the reference to the attitude subsystem which, in turn, affects the position error dynamics (see Figure 1).

The attitude planner design plays a central role in our control strategy. This subsystem takes the desired attitude reference and the position errors as inputs and is in charge of computing a reference attitude and angular velocity that are feasible, in the sense that the actuation constraints are satisfied. Specifically, the output of the planner (which is the actual reference to the attitude subsystem) is denoted as $\left(R_{p}, \omega_{p}\right) \in T \mathrm{SO}(3)$.

Property 1: The attitude planner output $\left(R_{p}, \omega_{p}\right) \in T \mathrm{SO}(3)$ is feasible, in the following sense

$$
\dot{R}_{p}(t)=R_{p}(t) \hat{\omega}_{p}(t), \quad \forall t \geq 0, \quad \omega_{p}(\cdot) \in C^{1} \cap L_{\infty} .
$$

\section{A. Control force and position error dynamics}

Since the goal of this work is to obtain a control law that prioritizes position over orientation tracking, we start by inspecting the position error dynamics. By focusing on the dynamics in (1)-(4), the natural choice of the tracking errors for position and velocity is:

$$
e_{x}:=x-x_{d}, \quad e_{v}:=v-v_{d} .
$$

Accordingly, the position and velocity errors are expressed in the inertial frame. The control objective is to stabilize the origin $\left(e_{x}, e_{v}\right)=(0,0) \in \mathbb{R}^{3} \times \mathbb{R}^{3}$. Consider the equations (1), (3) and the definition of the errors in (10). Then,

$$
\begin{aligned}
\dot{e}_{x} & =e_{v} \\
m \dot{e}_{v} & =m\left(\dot{v}-\dot{v}_{d}\right)=-m\left(\dot{v}_{d}+g e_{3}\right)+R f_{c} .
\end{aligned}
$$


The system would be fully actuated if one could arbitrarily assign $f_{c}$, and it would be possible to enforce $R f_{c}=f_{d}$, with

$$
f_{d}\left(e_{x}, e_{v}, \dot{v}_{d}\right):=\beta\left(e_{x}, e_{v}\right)+m\left(\dot{v}_{d}+g e_{3}\right),
$$

where $\beta: \mathbb{R}^{3} \times \mathbb{R}^{3} \rightarrow \mathbb{R}^{3}$ is a static state feedback stabilizer. The corresponding closed-loop dynamics would be described by (11) and $m \dot{e}_{v}=\beta\left(e_{x}, e_{v}\right)$. Thus, the origin would be globally asymptotically stable. In this work we will adopt the same strategy used in [17], corresponding to a nested saturations-based static nonlinear feedback:

$$
\beta\left(e_{x}, e_{v}\right):=\lambda_{2} \text { sat }\left(\frac{k_{2}}{\lambda_{2}}\left(e_{v}+\lambda_{1} \text { sat }\left(\frac{k_{1}}{\lambda_{1}} e_{x}\right)\right)\right),
$$

where $\operatorname{sat}(\cdot)$ is a smooth decentralized unit saturation function satisfying the properties mentioned in [Sec. II-A] [17], $k_{1}, k_{2}$ and $\lambda_{1}, \lambda_{2}$ are suitably chosen gains and saturation levels to be selected according to the guidelines of [Prop. 1] [17]. While we develop our theory for this specific stabilizer, we emphasize that similar generalizations to those reported in [11], [18] are possible, thus allowing for stabilizing laws inducing improved transients.

To account for the fact that it is not possible to obtain $R f_{c}=f_{d}$ when the control force cannot span $\mathbb{R}^{3}$, it is convenient to rewrite the velocity error dynamics as:

$$
\begin{aligned}
m \dot{e}_{v} & =-m\left(\dot{v}_{d}+g e_{3}\right)+R R_{p}^{T} R_{p} f_{c} \\
& =-m\left(\dot{v}_{d}+g e_{3}\right)+R_{e} R_{p} f_{c} .
\end{aligned}
$$

where $R_{p}$ is the reference attitude given by the attitude planner and

$$
R_{e}:=R R_{p}^{T} \in \mathrm{SO}(3)
$$

is the so-called left attitude error [1]. Introducing the corresponding angular velocity error,

$$
e_{\omega}:=\omega-\omega_{p},
$$

a natural choice for the control force $f_{c}$ is

$$
f_{c}:=R_{p}^{T} \Phi\left(R_{e}, e_{\omega}, t\right) f_{d},
$$

where $\Phi\left(R_{e}, e_{\omega}, t\right): T \mathrm{SO}(3) \times \mathbb{R}_{\geq 0} \rightarrow \mathbb{R}^{3 \times 3}$ is a design function, selected later, ensuring that $\Phi\left(R_{e}, e_{\omega}, t\right) \rightarrow I_{3}$ for $\left(R_{e}, e_{\omega}\right) \rightarrow$ $\left(I_{3}, 0\right)$ and $\forall t \geq 0$. Indeed, by adding and subtracting $f_{d}$ in equation (16), the closed-loop velocity error dynamics reads:

$$
m \dot{e}_{v}=\beta\left(e_{x}, e_{v}\right)+\Delta R\left(R_{e}, e_{\omega}, t\right) f_{d}\left(e_{x}, e_{v}, \dot{v}_{d}\right)
$$

where

$$
\Delta R\left(R_{e}, e_{\omega}, t\right):=R_{e} \Phi\left(R_{e}, e_{\omega}, t\right)-I_{3} .
$$

Written in this form, the position error is clearly affected by the attitude error through the term $\Delta R f_{d}$, which is the mismatch between the desired force $f_{d}$ and the actual control force resolved in the inertial frame, i.e., $R f_{c}$.

Remark 1: The control force is obtained by scaling the desired force $f_{d}$ by a suitable term $\Phi\left(R_{e}, e_{\omega}, t\right)$ dependent on the attitude error, and then by applying a rotation $R_{p}^{T}$ to the resulting vector. The idea behind the proposed control law is twofold. When the attitude error is large, the scaling term $\Phi\left(R_{e}, e_{\omega}, t\right)$ can be used to reduce the control force and, correspondingly, the overshoot in the position tracking is limited and the transient behavior can be improved. The second point is related to the fact that the desired attitude may be such that the constraints on $f_{c}$ expressed by equations (5)-(6) are still not respected with the control force defined in equation (19). However, by prioritizing position over orientation tracking one can consider the planner rotation matrix $R_{p}$ as an additional degree of freedom to ensure that the scaled control force $\Phi\left(R_{e}, e_{\omega}, t\right) f_{d}$ is eventually rotated to be compliant with the actuation constraint.

The rationale behind the proposed control law is that if the attitude error dynamics can be made asymptotically stable, then, for $t \rightarrow \infty, R_{e}(t) \rightarrow I_{3}, \Phi\left(R_{e}(t), e_{\omega}(t), t\right) \rightarrow I_{3}$ and the control force delivered in the inertial frame $R(t) f_{c}(t)=$
$R_{e}(t) \Phi(t) f_{d}(t) \rightarrow f_{d}(t)$, which is the force in the inertial frame required to track the desired position trajectory. Indeed, in this case, the mismatch between the desired and actual control force converges to zero as well, namely $\Delta R(t) f_{d}(t) \rightarrow 0$. Firstly, the choice of $\Phi$ must be such that the following property holds true for the attitude mismatch $\Delta R$.

Property 2: (Vanishing perturbations). Consider $\Delta R$ defined in (21). Given $V_{a}\left(R_{e}, e_{\omega}\right):=\sqrt{\left\|e_{\omega}\right\|^{2}+\left\|R_{e}\right\|_{\mathrm{SO}(3)}^{2}}$, there exists a bounded class- $\mathcal{K}$ function $\gamma(\cdot)$, satisfying $\forall\left(R_{e}, e_{\omega}, t\right) \in$ $T \mathrm{SO}(3) \times \mathbb{R}_{\geq 0}$ :

$$
\left\|\Delta R\left(R_{e}, e_{\omega}, t\right)\right\| \leq \gamma\left(V_{a}\left(R_{e}, e_{\omega}\right)\right) .
$$

We will now show a possible selection of $\Phi$ such that the above property is verified. A wide range of performance-oriented alternative choices are possible as long as they satisfy Property 2, which is needed to ensure that, at convergence, the magnitude of the delivered control force $f_{c}$ converges to the nominal force $f_{c}^{s s}(7)$. It is straightforward to employ a scaling transformation, dependent on the attitude error alone, as follows:

$$
\Phi\left(R_{e}, e_{\omega}, t\right):=c\left(R_{e}, t\right) I_{3},
$$

where $c: \mathrm{SO}(3) \times \mathbb{R}_{\geq 0} \rightarrow \mathbb{R}$ is a properly selected function. The next proposition, whose proof is given in [10, Appendix], gives an example of such scaling function, which is naturally written in terms of the angle $\theta_{e}$ between the desired direction $b_{p_{3}}:=R_{p} e_{3}$ and the vertical body axis $b_{3}:=R_{e_{3}}$ :

$$
\theta_{e}\left(R_{e}, R_{p}\right):=\arccos \left(b_{p_{3}}^{T} b_{3}\right)=\arccos \left(e_{3}^{T} R_{p}^{T} R_{e} R_{p} e_{3}\right) .
$$

Proposition 1: Given $c\left(R_{e}, t\right):=\frac{\ell-\left(1-\cos \left(\theta_{e}\left(R_{e}, R_{p}(t)\right)\right)\right)}{\ell}$ where $\theta_{e}$ is defined in (24), then, for $\Phi\left(R_{e}, e_{\omega}, t\right):=c\left(R_{e}, t\right) I_{3}$ and $\ell>2$, Property 2 is satisfied.

\section{B. Control torque and attitude error dynamics}

The attitude controller has to ensure the convergence of the attitude tracking errors according to the fully actuated rotational dynamics in equations (2), (4). By using matrix multiplication as the group operation, the attitude error $R_{e}=R R_{p}^{T}$, is employed as the attitude error measure in $\mathrm{SO}(3)$, which was already introduced in equation (17). The error kinematics are derived from (2) and (9) as follows:

$$
\dot{R}_{e}=\dot{R} R_{p}^{T}+R \dot{R}_{p}^{T}=R_{e} R_{p} \hat{e}_{\omega} R_{p}^{T} .
$$

Consider the system defined in (2), (4) and the control law

$$
\tau_{c}:=-R_{p}^{T} e_{R}-K_{\omega} e_{\omega}+J \dot{\omega}_{p}+\hat{\omega}_{p} J \omega,
$$

where $K_{\omega} \in \mathbb{R}^{3 \times 3}$ is positive definite and $e_{R}:=\operatorname{skew}\left(K_{R} R_{e}\right)^{\vee} \in$ $\mathbb{R}^{3}$ is the left-trivialized derivative of the modified trace function $\Psi_{K_{R}}\left(R_{e}\right):=\frac{1}{2} \operatorname{tr}\left(K_{R}\left(I-R_{e}\right)\right)$. The map $(\cdot)^{\vee}: \mathfrak{s o}(3) \mapsto \mathbb{R}^{3}$ used in the definition of $e_{R}$ represents the inverse of the $\hat{\imath}$ map defined in the notation section. It is assumed that matrix $K_{R} \in \mathbb{R}^{3 \times 3}$ is symmetric and that it satisfies:

$$
\operatorname{tr}\left(K_{R}\right) I_{3}-K_{R} \in \mathbb{R}_{>0}^{3 \times 3} .
$$

Using (25) and the control torque (26) with the rotational equations of motion (2), (4), the dynamics of the errors (17) and (18) reads:

$$
\begin{aligned}
\dot{R}_{e} & =R_{e} R_{p} \hat{e}_{\omega} R_{p}^{T} \\
J \dot{e}_{\omega} & =-R_{p}^{T} e_{R}-K_{\omega} e_{\omega}-\hat{e}_{\omega} J e_{\omega}-\hat{e}_{\omega} J \omega_{p} .
\end{aligned}
$$

The control torque (26), first proposed by [1], has a simpler expression than the one based on the right group error considered in [13] and no cancellation of non-harmful nonlinearities occurs. The equilibria for the attitude subsystem are the points where the differential of $\Psi_{K_{R}}$ and the angular velocity error vanish, i.e., $e_{R}=0, e_{\omega}=0$. The set of equilibria contains the desired equilibrium $\left(R_{e}, e_{\omega}\right)=\left(I_{3}, 0\right)$ and additional undesired configurations corresponding to the other critical points of $\Psi_{K_{R}}$. Due 
to this intrinsic structure of $\mathrm{SO}(3)$, no time-invariant continuous control law can globally stabilize $\left(I_{3}, 0\right)$. Nonetheless, by defining the scalar (positive from (27)):

$$
\ell_{R}:=\lambda_{m}\left(\operatorname{tr}\left(K_{R}\right) I_{3}-K_{R}\right)>0,
$$

it is well known that in the sublevel set

$$
\mathcal{S}_{R}:=\left\{R_{e} \in \mathrm{SO}(3): \Psi_{K_{R}}\left(R_{e}\right)<\ell_{R}\right\},
$$

the point $R_{e}=I_{3}$ is the unique critical point and minimum of $\Psi_{K_{R}}$. Next, the total error energy function

$$
V_{R}\left(R_{e}, e_{\omega}\right):=\frac{1}{2} e_{\omega}^{T} J e_{\omega}+\Psi_{K_{R}}\left(R_{e}\right),
$$

will be used in the stability analysis. It can be shown that this function is a Lyapunov candidate for the attitude error dynamics, i.e., it is positive definite about $\left(R_{e}, e_{\omega}\right)=\left(I_{3}, 0\right)$, continuously differentiable and radially unbounded in the direction of $\left\|e_{\omega}\right\| \rightarrow$ $\infty$. The following theorem, the proof of which is reported in Section VII to avoid breaking the flows of the exposition, establishes desirable properties of the attitude stabilizer.

Theorem 1: Consider the system described by (28)-(29) and a reference attitude $t \mapsto\left(R_{p}(t), \omega_{p}(t)\right) \in T \mathrm{SO}(3)$ satisfying Property 1 . For any symmetric matrix $K_{R} \in \mathbb{R}^{3 \times 3}$ satisfying (27) and any matrix $K_{\omega} \in \mathbb{R}_{>0}^{3 \times 3}$, the equilibrium point $\left(R_{e}, e_{\omega}\right)=$ $\left(I_{3}, 0\right)$ is uniformly asymptotically stable (UAS) with basin of attraction containing the set

$$
S_{a}:=\left\{\left(R_{e}, e_{\omega}\right) \in \operatorname{TSO}(3): V_{R}\left(R_{e}, e_{\omega}\right)<\ell_{R}\right\}
$$

where $\ell_{R}$ and $V_{R}$ are defined in (30) and (32).

Remark 2: By choosing $K_{R}=k_{R} I_{3}, k_{r} \in \mathbb{R}_{>0}$, which satisfies (30) with $\ell_{R}=2 k_{R}$, the sublevel set of $\Psi_{K_{R}}$ in (31) contains all the rotations with an angle strictly less than $180^{\circ}$, namely almost all the configurations in $\mathrm{SO}(3)$. By increasing the gain $k_{R}$, also the set of initial conditions $e_{\omega}\left(t_{0}\right)$ included in (33) can be arbitrarily enlarged achieving a semi-global stability property. Robust global asymptotic stability of the desired equilibrium $\left(I_{3}, 0\right)$ can be obtained by using a hybrid controller on $\mathrm{SO}(3)$ [15].

Remark 3: As an alternative choice to (26), the control law $\tilde{\tau}_{c}:=$ $\hat{\omega} J \omega+J\left(\dot{\omega}_{p}-\hat{\omega}_{p} \omega-R_{p}^{T}\left(e_{R}+K_{\omega} R_{p} e_{\omega}\right)\right)$ substituted in (2), (4) provides the following closed-loop:

$$
\begin{aligned}
\dot{R}_{e} & =R_{e} \hat{\tilde{e}}_{\omega} \\
\dot{\tilde{e}}_{\omega} & =-e_{R}-K_{\omega} \tilde{e}_{\omega},
\end{aligned}
$$

where $\tilde{e}_{\omega}:=R_{p}\left(\omega-\omega_{p}\right)$. In this way the closed loop is autonomous and independent of $\omega_{p}$. Even though this choice would make the stability analysis of the control scheme simpler and would place less restrictions on the position stabilizer (see Remark 6), it would also make the design less general. For instance, the feedback $\tilde{\tau}_{c}$ cancels non-harmful nonlinearities making it less desirable from the point of view of actuation effort.

\section{Complete dynamics}

This section presents the main results of the stability analysis for the complete system. Our proof is based on a compact representation of the closed loop wherein the solutions of the time-varying dynamics is embedded into a time-invariant differential inclusion, in ways that are similar to the strategy in [17], even though the approach adopted here does not require the (somewhat stringent) assumption $\dot{\omega}_{p}$ be bounded. By introducing $x_{a}:=\left(R_{e}, e_{\omega}\right)$ and $x_{p}:=\left(e_{x}, e_{v}\right)$, the solutions to the attitude error dynamics (28)(29) and the position error dynamics (11), (20) can be embedded within the solution funnel generated by the following constrained differential inclusion ${ }^{1}$ :

$$
\begin{aligned}
& \text { (A) } \dot{x}_{a} \in F_{a}\left(x_{a}\right), \quad x_{a} \in T \operatorname{TSO}(3) \\
& \text { (P) } \quad \dot{x}_{p} \in F_{p}\left(x_{p}, x_{a}\right), \quad x_{p} \in \mathbb{R}^{3} \times \mathbb{R}^{3},
\end{aligned}
$$

with a slight abuse of notation ${ }^{2}$ and where $F_{a}\left(x_{a}\right), F_{p}\left(x_{p}\right)$ are

$$
\begin{aligned}
& F_{a}\left(x_{a}\right):=
\end{aligned}
$$

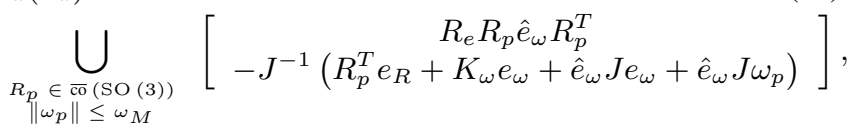

$$
\begin{aligned}
& F_{p}\left(x_{p}, x_{a}\right):=\left[\begin{array}{c}
e_{v} \\
\frac{1}{m}\left(\beta\left(e_{x}, e_{v}\right)+f_{M} \gamma\left(V_{a}\left(R_{e}, e_{\omega}\right)\right) \overline{\mathcal{B}_{3}}\right)
\end{array}\right]
\end{aligned}
$$

where $\overline{\mathrm{co}}(\cdot)$ denotes the closed convex hull, $\overline{\mathcal{B}_{3}}$ is the closed unit ball and $\omega_{M} \in \mathbb{R}_{>0}$ is a constant existing thanks to Assumption 1. Moreover, function $\gamma$ comes from (22), and scalar $f_{M}:=\sqrt{3} \lambda_{2}+$ $f_{M}^{s s}$ is a bound on $f_{d}$ arising from substituting (7) and (40) into (13).

Based on representation (36), (37), asymptotic tracking for the complete dynamics can be proven, under Assumption 1, as stated by the following Theorem.

Theorem 2: Consider the closed loop system described by (11), (20) and (28), (29) controlled by (19), (26) and the planner output given by $\left(R_{p}, \omega_{p}\right)=\left(R_{d}, \omega_{d}\right) \in T \mathrm{SO}(3)$, where the desired trajectory $t \mapsto\left(R_{d}(t), \omega_{d}(t), x_{d}(t), v_{d}(t)\right)$ satisfies Assumption 1 . Then, if $\Phi(\cdot, \cdot, \cdot)$ is selected according to Property 2, for any symmetric matrix $K_{R}$ satisfying (27), any $K_{\omega} \in \mathbb{R}_{>0}^{3 \times 3}$, the point $\left(R_{e}, e_{\omega}, e_{x}, e_{v}\right)=\left(I_{3}, 0,0,0\right)$ is robustly uniformly asymptotically stable with basin of attraction containing the set $S_{a} \times \mathbb{R}^{3} \times \mathbb{R}^{3}$, where $S_{a}$ is defined in (33).

Proof: The cascaded interconnection (36), (37) comprises the upper subsystem (36), whose stability properties (with domain of attraction $S_{a}$ ) is established in Theorem 1, and the lower subsystem (37), which is stabilized by the nested saturation feedback (14) proposed in [17]. (Local) stability of the cascade follows from standard reduction theorems for differential inclusions (see, e.g., [14]) whereas attractivity from $S_{a} \times \mathbb{R}^{3} \times \mathbb{R}^{3}$ can be established using the small signal ISS properties of stabilizer (14) following the same steps as in [17, Proof of Prop. 4]. Finally, stability and attractivity of the point $\left(R_{e}, e_{\omega}, e_{x}, e_{v}\right)=\left(I_{3}, 0,0,0\right)$ for the closed loop implies robust uniform asymptotic stability from $S_{a} \times \mathbb{R}^{3} \times \mathbb{R}^{3}$, by concatenating the implications in [6, Thm 7.12] and [6, Thm 7.21].

\section{ATtitude PLANNING FOR CONSTRAINTS COMPLIANCE}

Theorem 2 establishes robust asymptotic stability of the origin for the error dynamics, regardless of the reference orientation $\left(R_{p}, \omega_{p}\right) \in T \mathrm{SO}(3)$. Nonetheless, it gives no guarantees about the fact that the force $f_{c}$ requested by the control scheme satisfies the constraints characterized in Section II. In this section we propose to select $\left(R_{p}, \omega_{p}\right)$ according to a dynamic attitude planner, as represented in Figure 1, which is in charge of properly changing the desired attitude to prioritize position over orientation tracking, while respecting the input constraints at hand. With respect to the approach of [5], in which the reference attitude is obtained as the solution of an optimization problem, our design allows to naturally compute a differentiable reference that satisfies Property 1 so that $\tau_{c}$ in (26) is well defined. Note also that the control design of [5], [8] limits the choice of attitude stabilizers to those making the attitude error subsystem autonomous and the desired equilibrium exponentially stable.

${ }^{1}$ Following [6, $\left.\S 6.2 .1\right]$, the regularity of the data of our constrained differential inclusions simplifies the definition of solutions of $\dot{x} \in F(x)$, $x \in C$, corresponding to any absolutely continuous function $\phi$ such that $\dot{\phi}(t) \in F(\phi(t))$ and $\phi(t) \in C$ for almost all $t$.

${ }^{2}$ To be consistent with the formulation, the differential inclusion should be written by exploiting the vectorization, $\operatorname{vec}\left(\dot{R}_{e}\right) \in \operatorname{vec}\left(R_{e} R_{p} \hat{e}_{\omega} R_{p}^{T}\right)$ 
Before proceeding with the planner design, we recall the following additional assumptions on the desired trajectory which are needed to ensure a well-defined planner reference at all times.

Assumption 2: The desired trajectory $t \mapsto\left(R_{d}(t), \omega_{d}(t), x_{d}(t)\right.$, $\left.v_{d}(t)\right) \in T \operatorname{SO}(3) \times \mathbb{R}^{3} \times \mathbb{R}^{3}$ satisfies Assumption 1 and

1) the desired acceleration $\dot{v}_{d}(\cdot)$ is such that the nominal force (7) is bounded by some strictly positive constants $f_{m}^{s s}, f_{m}^{s s}$, i.e.,

$$
0<f_{m}^{s s} \leq\left\|f_{c}^{s s}(t)\right\| \leq f_{M}^{s s}<T_{M} \quad \forall t \geq 0
$$

and that $\inf _{t \geq 0}\left(m\left|g+\dot{v}_{d_{3}}\right|\right)>0$;

2) the desired trajectory $x_{d}(\cdot)$ belongs at least to $C^{4}$;

3) the desired jerk $\ddot{v}_{d}(\cdot)$ belongs to $L_{\infty}$

\section{A. Attitude planner dynamics}

There is a natural way to express the reference attitude $R_{p}$ by noticing that the actuation constraints for all the different configurations are satisfied if the control force is delivered along the positive direction of the $b_{3}$ axis. Following standard strategies for underactuated UAVs [13], we introduce a smooth matrix function $R_{c}\left(f_{d}, t\right): \mathbb{R}^{3} \backslash\{0\} \times \mathbb{R}_{\geq 0} \rightarrow \mathrm{SO}(3)$ defined as:

$$
R_{c}\left(f_{d}, t\right):=\left[\begin{array}{lll}
b_{c_{1}}\left(f_{d}, t\right) & b_{c_{2}}\left(f_{d}, t\right) & b_{c_{3}}\left(f_{d}\right)
\end{array}\right]
$$

where $b_{c_{3}}:=f_{d} /\left\|f_{d}\right\|$ and $b_{c_{1}}\left(f_{d}, t\right)$ and $b_{c_{2}}\left(f_{d}, t\right)$ are any two orthogonal unit vectors such that $R_{c}$ defines a rotation matrix. A possible selection is:

$$
b_{c_{1}}:=b_{c_{2}}\left(f_{d}, t\right) \times b_{c_{3}}\left(f_{d}\right), \quad b_{c_{2}}:=\frac{b_{c_{3}}\left(f_{d}\right) \times b_{d}(t)}{\left\|b_{c_{3}}\left(f_{d}\right) \times b_{d}(t)\right\|} .
$$

The vector $b_{d}$ defines the desired heading direction of the UAV:

$$
b_{d}(t):=\left[\begin{array}{lll}
\cos \left(\psi_{d}(t)\right) & \sin \left(\psi_{d}(t)\right) & 0
\end{array}\right]^{T},
$$

where $t \mapsto \psi_{d}(t) \in \mathbb{R}$ is the desired yaw angle (which may be extracted from a given $R_{d}(t)$ ). We note that $R_{c}\left(f_{d}, t\right)$ is well defined as long as $f_{d} \neq\left[\begin{array}{lll}0 & 0 & 0\end{array}\right]^{T}$. Based on $R_{c}$, we select the reference attitude $R_{p}$, output of the attitude planner, as:

$$
R_{p}:=R_{c}\left(f_{d}, t\right) R_{r},
$$

where $R_{r} \in \mathrm{SO}(3)$ is an additional state of the dynamic attitude planner. Being an element of $\mathrm{SO}(3)$, the differential equation for the relative attitude $R_{r}$ can be written as:

$$
\dot{R}_{r}=\hat{\omega}_{r} R_{r},
$$

where $\omega_{r} \in \mathbb{R}^{3}$ is the relative angular velocity, with coordinates in the frame $\mathcal{F}_{c}$, that will be used as an auxiliary input to control the evolution of $R_{r}$. For instance, $\omega_{r}$ can be used to track at best the desired attitude $R_{d}$ by exploiting a Lyapunov-based design, once a suitable potential function of the desired attitude $R_{d}$ is provided. Then, the time evolution of $R_{r}$ can be properly modified in order to satisfy the actuation constraints (see Section VI). Finally, the attitude planner has to provide a corresponding velocity reference $\omega_{p}$, satisfying $R_{p}=R_{p} \hat{\omega}_{p}$, which is computed by first introducing the angular velocity

$$
\omega_{c}\left(R_{c}, f_{d}\right):=\left(R_{c}^{T} \dot{R}_{c}\right)^{\vee}
$$

of the frame $\mathcal{F}_{C}:=\left\{b_{c_{1}}, b_{c_{2}}, b_{c_{3}}\right\}$, and then using (44) and (45) to obtain $\dot{R}_{p}=\dot{R}_{c} R_{r}+R_{c} \dot{R}_{r}=R_{p} R_{r}^{T}\left(\hat{\omega}_{c}+\hat{\omega}_{r}\right) R_{r}=R_{p} \hat{\omega}_{p}$. In particular, the above relation provides $\hat{\omega}_{p}=R_{r}^{T}\left(\hat{\omega}_{c}+\hat{\omega}_{r}\right) R_{r}$ which, by exploiting the vee $(\cdot)^{\vee}$ map gives:

$$
\omega_{p}=R_{r}^{T}\left(\omega_{c}\left(R_{c}, f_{d}\right)+\omega_{r}\right)
$$

As the main goal of the attitude planner is to track at best the desired attitude, it is more convenient to rewrite the dynamics (45) in terms of the planner attitude error, i.e.,

$$
R_{e}^{p}:=R_{p} R_{d}^{T}
$$

Using then $\dot{R}_{d}=R_{d} \hat{\omega}_{d}$ from Assumption 1 and (47), the overall dynamics of the attitude planner becomes:

$$
\begin{aligned}
\dot{R}_{e}^{p} & =R_{e}^{p} R_{d}\left(\hat{\omega}_{p}-\hat{\omega}_{d}\right) R_{d}^{T} \\
\omega_{p} & =\left(R_{c}^{T} R_{e}^{p} R_{d}\right)^{T}\left(\omega_{c}\left(R_{c}, f_{d}\right)+\omega_{r}\right) .
\end{aligned}
$$

Proposition 2: By selecting the gain $\lambda_{2}<\inf _{t \geq 0} \mid m\left(\dot{v}_{d_{3}}(t)+\right.$ $g) \mid$ in (14), the rotation matrix $R_{c}$ by (41)-(43), is well defined when the desired trajectory satisfies Assumption 2.

Proof: The proposition can be proven by inspecting the following inequality:

$$
\begin{array}{r}
\left|\left\|f_{d}(t)\right\| \geq\right| f_{d_{3}}(t)|\geq m| \dot{v}_{d_{3}}(t)+g|-| \beta_{3}\left(e_{x}, e_{v}\right) \mid \\
\geq \inf _{t \geq 0}\left|m\left(\dot{v}_{d_{3}}(t)+g\right)\right|-\beta_{3_{M}}>0,
\end{array}
$$

which holds thanks to the equivalence $\beta_{3_{M}}=\lambda_{2}$ for the definition of (14) coming from [17] and the assumption that $\lambda_{2}<$ $\inf _{t \geq 0}\left|m\left(\dot{v}_{d_{3}}(t)+g\right)\right|$. Then, $b_{c_{1}}$ and $b_{c_{2}}$ are unit vectors, orthogonal to each other and with $b_{c_{3}}$ and well defined $\forall t \geq 0$. Indeed, $b_{d}(t)$ belongs to the horizontal plane by definition $(43)$ and the third component of $b_{c_{3}}$ never vanishes from (51). Hence, the cross product $b_{c_{3}} \times b_{d}$ does not vanish either and so $\left\|b_{c_{3}} \times b_{d}\right\| \neq 0$ $\forall t \geq 0$ in equation (42).

The following lemma is a useful link between the output $\left(R_{p}, \omega_{p}\right)$ of the attitude planner, and the reference motion satisfying the properties of Assumption 1. Its proof is given in [10, Appendix].

Lemma 1: (Feasibility conditions of the planner output). If the relative angular velocity is bounded and continuously differentiable, i.e., $\omega_{r} \in\left(L_{\infty} \cap C^{1}\right)$, and the desired angular velocity $\omega_{d}$ satisfies Assumption 1, then the reference attitude motion $\left(R_{p}, \omega_{p}\right) \in$ $T S O(3)$, obtained according to equations (44), (47), is feasible, in the sense that it satisfies Property 1.

Remark 4: The decomposition of the reference attitude in equation (44) allows to effectively account for the actuation constraints of the different configurations. The angular velocity $\omega_{r}$ of the relative rotation matrix is an additional degree of freedom that can be exploited when the thrust-vectoring capability is not locked, i.e., the control force can be produced in a region around the vertical axis. This additional input can be exploited to track the desired attitude at best while taking into account the constraints. Indeed, it is always possible to select an initial condition $R_{r}(0)$ such that the actuation constraints are verified. Then, the evolution of the relative attitude can be properly controlled by modifying the angular velocity input $\omega_{r}$ to satisfy the constraints.

Remark 5: The requirement $x_{d}(\cdot) \in C^{4}$ in Assumption 1 allows us to properly define the time derivative of $\omega_{p}$, which is required to apply the attitude control law, as shown in the next section. Indeed, by direct computation from (47):

$$
\dot{\omega}_{p}=-R_{r}^{T} \hat{\omega}_{r} \omega_{c}+R_{r}^{T}\left(\dot{\omega}_{c}+\dot{\omega}_{r}\right),
$$

where $\dot{\omega}_{c}=\left(R_{c}^{T} \ddot{R}_{c}-\hat{\omega}_{c}^{2}\right)^{\vee}$.

\section{B. Special selections}

In this section we illustrate the relevance of the proposed control scheme for addressing the input limitations of thrust-vectoring UAVs (case (b) ). A similar analysis about vectored-thrust configurations (case (a)) can be found in [10], [11].

Within the present design, we make use of the relative rotation matrix $R_{r}$ introduced in (44) to perform attitude maneuvers that are compatible with position tracking. By considering that $R_{c}$ defined in (41) satisfies $R_{c} e_{3}=\frac{f_{d}}{\left\|f_{d}\right\|}$, the control force can be written as:

$f_{c}=c\left(R_{e}, t\right) R_{r}^{T} R_{c}^{T} f_{d}=c\left(R_{e}, t\right) R_{r}^{T}\left\|f_{d}\right\| e_{3}=c\left(R_{e}, t\right)\left\|f_{d}\right\| R_{r}^{T} e_{3}$.

By substituting (54) into the constraint (6), we get:

$$
\cos \left(\theta_{c}\right)=\frac{c\left(R_{e}, t\right)\left\|f_{d}\right\| R_{r}^{T} e_{3}}{c\left(R_{e}, t\right)\left\|f_{d}\right\|}=e_{3}^{T} R_{r} e_{3}=e_{3}^{T} b_{r_{3}},
$$


which shows that in order to satisfy (6), it is sufficient to guarantee that $e_{3}^{T} b_{r_{3}} \geq \cos \left(\theta_{M}\right)$. In this section we will show how the solution proposed in [12] to compute the relative angular velocity $\omega_{r}$ of equation (45) to account for the conic region constraint (19) and exploit the thrust-vectoring capabilities of tiltrotor configurations can be applied within the present framework. In particular, we will verify that the planner output obtained by selecting

$$
\omega_{r}=b_{r_{3}} \times \operatorname{Proj}_{G}\left(\omega_{r}^{d} \times b_{r_{3}}\right)+\left(b_{r_{3}}^{T} \omega_{r}^{d}\right) b_{r_{3}},
$$

where $\operatorname{Proj}_{G}: \mathbb{R}^{3} \rightarrow \mathbb{R}^{3}$ is a geometric projection operator ${ }^{3}$ and

$$
\omega_{r}^{d}=R_{r} \omega_{d}-\omega_{c}-R_{r} R_{d}^{T} e_{R}^{p}
$$

with $e_{R}^{p}:=k_{d} \operatorname{skew}\left(R_{e}^{p}\right)^{\vee}$, satisfies Property 1 . The projection operator keeps the planar vector $b_{r_{3}}^{\perp}:=\left[e_{1}^{T} b_{r_{3}} e_{2}^{T} b_{r_{3}}\right]$ inside a circle of radius $\delta:=\sin \left(\theta_{M}\right)$ by acting on the vector field $\omega_{r}^{d}$ in the region $\left(\frac{\delta}{\sqrt{1+\varepsilon}}, \delta\right]$, where $\varepsilon \in(0,1)$ is a user-defined parameter. In particular, thanks to the projection operator, $e_{3}^{T} b_{r_{3}}(t) \geq \cos \left(\theta_{M}\right)$ $\forall t \geq 0$ and, by virtue of (55), also:

$$
\cos \left(\theta_{c}(t)\right) \geq \cos \left(\theta_{M}\right) \quad \forall t \geq 0 .
$$

As a consequence, if the relative attitude is initialized such that $b_{r_{3}}$ is inside a cone defined by $\theta_{M}$ around $b_{c_{3}}$, it will never leave it.

Lemma 2: The relative angular velocity computed according to equation (56) is such that the planner output $\left(R_{p}, \omega_{p}\right) \in T \mathrm{SO}(3)$ obtained with (44), (47), satisfies Lemma 1.

Proof: By exploiting a smooth Projection operator, $\omega_{r}$ defined in (56) is $C^{1}$ and its time derivative can be computed as in (53). Then, we can write the following bound on $\omega_{r}$ :

$$
\left\|\omega_{r}\right\| \leq\left\|\operatorname{Proj}_{G}\left(\omega_{r}^{d} \times b_{r_{3}}\right)\right\|+\left\|\omega_{r}^{d}\right\|
$$

Notice that the projection operator simply removes the radial component of $\dot{b}_{r_{3}}^{d}$, when it is working, hence $\left\|\operatorname{Proj}_{G}\left(\omega_{r}^{d} \times b_{r_{3}}\right)\right\| \leq$ $\left\|\omega_{r}^{d}\right\|$ because $b_{r_{3}}$ is a unit vector. Finally, we can conclude the boundedness of $\omega_{r}^{d}$ from the following inequality:

$$
\left\|\omega_{r}^{d}\right\| \leq\left\|\omega_{d}\right\|+\left\|\omega_{c}\right\|+\left\|e_{R}^{p}\right\|
$$

in which $\omega_{d}$ is bounded according to Assumption 1, $\omega_{c}$ is bounded as shown in [10, Appendix], and $e_{R}^{p}$ is bounded, being the lefttrivialized derivative of a function defined on a compact manifold.

Definition 1: A trajectory $t \mapsto\left(R_{d}(t), \omega_{d}(t), x_{d}(t), v_{d}(t)\right) \in$ $T \mathrm{SO}(3) \times \mathbb{R}^{3} \times \mathbb{R}^{3}$ satisfying Assumption 2 is compatible with the position tracking task for the system of case (b) if, given $\delta:=$ $\sin \left(\theta_{M}\right), \varepsilon \in(0,1)$ and $\theta_{b}:=\arcsin \left(\frac{\delta}{\sqrt{1+\epsilon}}\right)$, there exists $\bar{t} \in$ $\mathbb{R}_{\geq 0}$, such that

$$
\frac{e_{3}^{T} f_{c}^{s s}(t)}{\left\|f_{c}^{s s}(t)\right\|} \geq \cos \left(\theta_{b}\right) \quad \forall t \geq \bar{t}
$$

where $f_{c}^{s s}(t)$ is defined in equation (7).

According to Definition 1, the attitude motion is compatible with the conic region constraint (6) if the angle between the third desired axis $b_{d_{3}}$ and the nominal force $R_{d} f_{c}^{s s}(t)$ is within the cone in which the $\operatorname{Proj}_{G}$ operator is not active. The next corollary combines the results of Theorem 2 and Lemma 2.

Corollary 1: Consider the closed loop system described by (11), (20), (28), (29), (49) controlled by (19), (26), where the planner output $\left(R_{p}, \omega_{p}\right)$ is given by (44) and (50), with $R_{c}, \omega_{c}$ and $\omega_{r}$ selected as in (41)-(43), (46) and (56)-(57), respectively. Assume that the desired trajectory $t \mapsto\left(R_{d}(t), \omega_{d}(t), x_{d}(t), v_{d}(t)\right)$ is trackable according to Definition 1 , that $\Phi(\cdot, \cdot, \cdot)$ is selected according to (23) and satisfies Property 2, and that the gains $k_{1}, k_{2}$ and saturation levels $\lambda_{1}, \lambda_{2}$ in (14) are selected according

\footnotetext{
${ }^{3}$ The geometric projection operator is an extension of the smooth projection operator for systems evolving on $\mathbb{R}^{n}$ originally proposed in [2], to the case of systems evolving on $\mathbb{S}^{n}$.
}

to [17, Prop. 1] and Proposition 2. Then, for any symmetric matrix $K_{R}$ satisfying $(27), K_{\omega} \in \mathbb{R}_{>0}^{3 \times 3}, \varepsilon \in(0,1)$ and $k_{d} \in$ $\mathbb{R}_{>0}$, the control force (19) satisfies the actuation constraint (6) and the point $\left(R_{e}, e_{\omega}, e_{x}, e_{v}, R_{e}^{p}\right)=\left(I_{3}, 0,0,0, I_{3}\right)$ is robustly asymptotically stable with basin of attraction containing the set $S_{a} \times \mathbb{R}^{3} \times \mathbb{R}^{3} \times S_{a p}$, where $S_{a}$ is given by (33) and $S_{a p}:=$ $\left\{R_{e}^{p} \in \mathrm{SO}(3): e_{3}^{T} R_{d}^{T}(0)\left(R_{e}^{p}\right)^{T} \frac{f_{d}(0)}{\left\|f_{d}(0)\right\|} \geq \cos \left(\theta_{M}\right)\right\}$, with $f_{d}$ defined in (13).

Proof: The proof is based on Theorem 2, Lemma 2 and [12, Thm 3]. Lemma 2 guarantees that the output planner reference $\left(R_{p}, \omega_{p}\right) \in T \mathrm{SO}(3)$ computed according to (44) and (56) satisfies Property 1 . Therefore, the assumptions of Theorem 2 are satisfied and the point $\left(R_{e}, e_{\omega}, e_{x}, e_{v}\right)=\left(I_{3}, 0,0,0\right)$ is UAS. Furthermore, because $R_{e}^{p}$ evolves on $\mathrm{SO}(3)$ according to (49), (50) with $\omega_{r}$ given by (56) and because the projection operator guarantees that equation (58) holds $\forall t \geq 0$, the first part of the theorem is demonstrated. Then, following the proof [12, Thm 3], combining the trackability condition in Definition 1 and the properties of the projection operator together with the expression of $\omega_{r}^{d}$ in (57), the theorem can be proved.

Remark 6: (Classes of position and attitude stabilizers). Any position stabilizer $\beta(\cdot, \cdot)$ satisfying the conditions of [Property 2] [11] would work in Corollary 1. At the same time, different selections of the control torque (see [1] for some alternatives beyond the one in Remark 3) could be considered as long as they guarantee UAS of $\left(R_{e}, e_{\omega}\right)=\left(I_{3}, 0\right)$ for any planner reference $t \mapsto\left(R_{p}(t), \omega_{p}(t)\right)$. Following Remark 3 , our selection in equation (26) is quite general in terms of characteristics of the attitude error subsystem that one has to consider when addressing the stability analysis of the complete control scheme. While this makes our stability analysis quite general, it comes at the expense of more stringent requirements on the position stabilizer (second item in [Property 2] [11]) to guarantee that the planner velocity is bounded (Property 1).

\section{NUMERICAL RESULTS}

The control law designed in the previous sections will be applied to the case of a hexarotor UAV in which each propeller is tilted by an angle $\alpha$ with respect to the local $x$-axis (see Figure 2). The mapping from $u:=\left(\omega_{1}, \ldots, \omega_{6}\right)$ to the control wrench $w_{c}$ can be compactly written as $w_{c}=M(\alpha) \bar{u}$ [10, eqn. 74], where $\omega_{i} \in\left[0, \omega_{M}\right]$ is the angular rate of the $i$-th propeller, $\bar{u}:=\left(\omega_{1}^{2}, \ldots, \omega_{6}^{2}\right)$ and $M(\alpha) \in \mathbb{R}^{6 \times 6}$ is a matrix dependent upon physical quantities of the UAV and the tilt angle $\alpha$. Note that nonfeasible, i.e., negative, angular rates for the individual propellers can be obtained when inverting $M(\alpha)$ for a given control wrench. This can be understood by considering that the force delivered according to $M(\alpha) \bar{u}$ spans only a given region in the space around $b_{3}$, the size of which is closely related to the value of $\alpha$. The model of case $(b)$ in Section II is reasonable to approximate the actuation constraint of the considered hexarotor.

The simulation model is a multi-body system with seven bodies (a central body and six propellers groups) written in the Modelica modeling language, which accounts for the actuators dynamics as well as aerodynamic effects. A conservative source of drag has been considered in the model to verify robustness, which is one of the main claims of Theorem 2. Furthermore, the control law has been implemented in a $500 \mathrm{~Hz}$ sample-data scenario [5] with a time delay of two samples, together with typical measurement noise experienced in indoor flight conditions with a motion capture system. The mass and inertial matrix of the UAV are $m=1 \mathrm{~kg}$ and $J=\operatorname{diag}(0.008,0.008,0.016) \mathrm{kg} \cdot \mathrm{m}^{2}$, respectively. By considering $\alpha=20^{\circ}$, the conic region (6) with $\theta_{M}=10^{\circ}$ was found to be a reasonable approximation of the wrench map $M(\alpha)$ to ensure its invertibility in a broad operational range. The controller gains are $K_{R}=\operatorname{diag}(0.6,0.6,1.4), K_{\omega}=0.2 I_{3}, \ell=2.1, k_{d}=2, \lambda_{2}=$ $9, \lambda_{1}=1, k_{1}=0.06 k_{2}=9, \varepsilon=0.05$. The desired position trajectory is a circle $x_{d}(t)=\left(\cos \left(\Omega_{d}(t) t\right), \cos \left(\Omega_{d}(t) t\right), 0\right) \mathrm{m}$, see 
Figure 2, where $\Omega_{d}(t)$ is made by two constant intervals connected by a smooth ramp (top of Figure 3). The desired attitude is $R_{d}(t)=$ $I_{3}$, i.e., the UAV has to fly with level attitude, a requirement that is never compatible with vectored-thrust configurations. The initial conditions correspond to hover at $x(0)=(1,0,0) \mathrm{m}$. By inspecting Figure 3 (bottom), the considered trajectory is not always feasible in the sense of Definition 1. Indeed, the inclination angle of the steady state control force (7) with respect to the vertical body axis, i.e., $\theta_{n}:=\arccos \left(e_{3}^{T} f_{c}^{s s}(t) /\left\|f_{c}^{s s}(t)\right\|\right)$, is greater than the maximum tilt angle $\theta_{M}$ in the interval $t \in[17,26] \mathrm{s}$. It is worth mentioning that $\theta_{n}$ represents also the inclination that a vectored-thrust UAV would have to reach to guarantee position tracking. Figure 4 shows that, after a transient phase, the position tracking errors remain bounded: aerodynamic drag prevents their actual convergence to zero. The oscillations shown at steady state are induced by the combination of aerodynamic drag (which works in the direction of $-v$ ) and the periodic nature of the circular motion. The attitude tracking performance is shown in Figure 5, where the inclination angle of the body axis $b_{3}$ with respect to the inertial axis $e_{3}$, i.e., $\theta_{v}:=\arccos \left(b_{3}^{T} e_{3}\right)$, is plotted (top) together with the yaw angle $\psi$ (bottom). Since the desired attitude is $R_{d}(t)=I_{3}$, the corresponding desired values are $\theta_{v}^{d}(t)=0^{\circ}$ and $\psi_{d}(t)=0^{\circ}$. Additional plots showing the control variables can be found in the extended report [10]. When the trajectory is feasible, the UAV is capable of flying at almost level attitude $\left(\theta_{v}(t) \approx 0.5^{\circ}\right)$. On the contrary, during the initial transient and the acceleration phase, when the desired attitude is not compatible with the thrust-vectoring constraint (6) and position tracking, the attitude tracking objective is only partially achieved and the projection operator is working to modify the attitude reference so that position tracking is guaranteed. Note from Figure 2 and Figure 5 that the vehicle is inclined of an angle $\theta_{v}=11.62^{\circ}$ at $\bar{t} \approx 21 \mathrm{~s}$ whereas the nominal angle is $\theta_{n}(\bar{t}) \approx 21^{\circ}>\theta_{M}$. Therefore, the proposed solution tries to stay as close as possible to the desired attitude $\left(\theta_{v}^{d}=0\right)$ even if the trajectory is not trackable. Furthermore, note that the primary objective is not affected during this phase: the position tracking performance is not deteriorated. It is worth mentioning that the tracking errors converge to zero (see Figure 4 and Figure 5, dashed lines) when the control model (CM) is employed in the simulation, as expected from Corollary 1. Finally, the conic region constraint (6) is satisfied at all times, i.e., $\theta_{c}(t) \leq \theta_{M}$, as shown in Figure 3 (bottom, solid line).

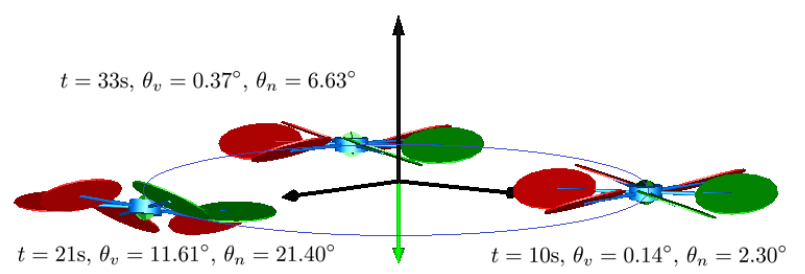

Fig. 2. Trajectory followed by the hexacopter.

\section{PRoOF OF THEOREM 1}

To prove Theorem 1, we write the attitude error dynamics as a constrained differential inclusion and then we apply an invariance principle to prove the asymptotic stability of the desired attractor. Equations (28), (29) describe the evolution of the attitude error dynamics, the solutions of which are also solutions of the differential inclusion $\dot{x}_{a} \in F_{a}$, where $F_{a}$, defined in (38), is clearly an outer semicontinuous (its graph is closed) and locally bounded set-valued map, and for each $x_{a} \in T \mathrm{SO}(3), F_{a}\left(x_{a}\right)$ is nonempty and convex. To prove Theorem 1 it will be convenient to intersect $F_{a}\left(x_{a}\right)$ with the tangent cone to $T \mathrm{SO}(3)$, denoted as $T_{T \mathrm{SO}(3)}\left(x_{a}\right)$ at $x_{a}$. Indeed the solutions to (36) can only flow along the directions in the tangent cone, which simplifies the Lyapunov analysis (see [20] for details). To this end, the following lemma is useful:
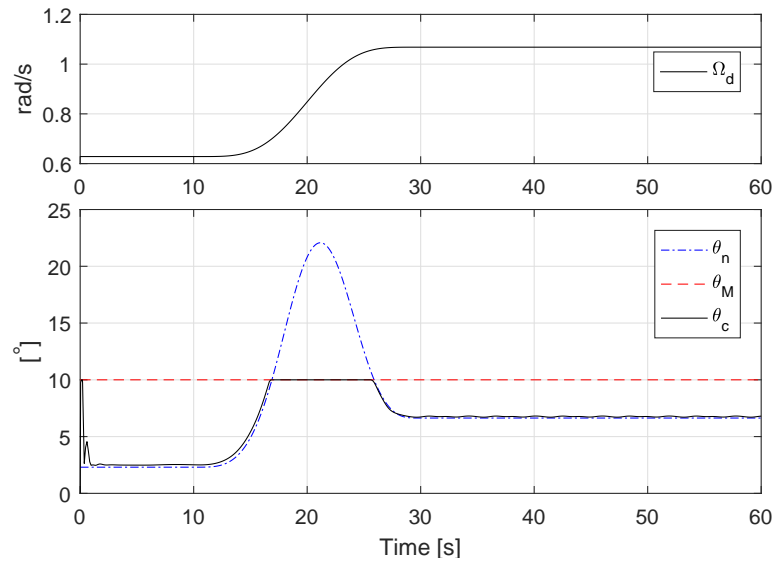

Fig. 3. $\Omega_{d}(t)$ (top) - Maximum tilt angle $\theta_{M}$, nominal angle $\theta_{n}$ of the steady state force with respect $b_{3}$ and control force angle $\theta_{c}$ (bottom).

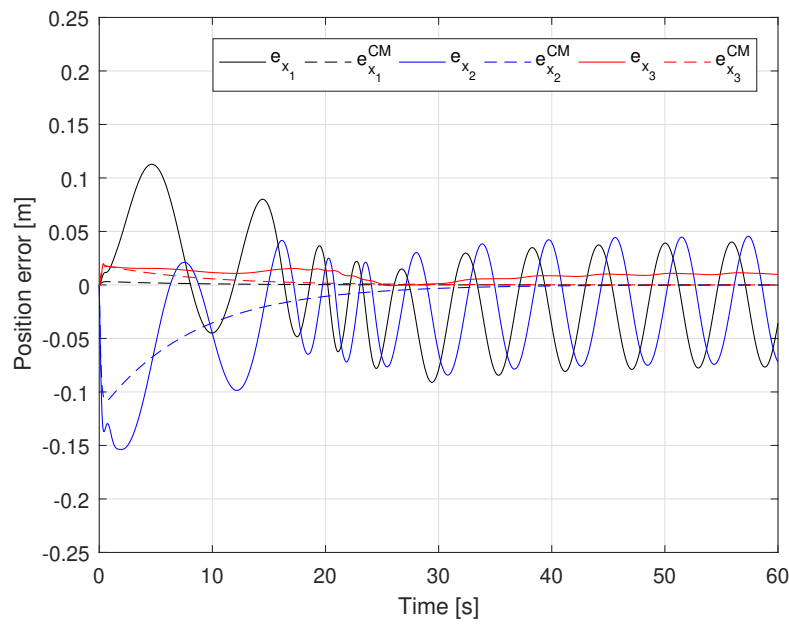

Fig. 4. Position tracking error $e_{x}$ (dashed lines: control model).
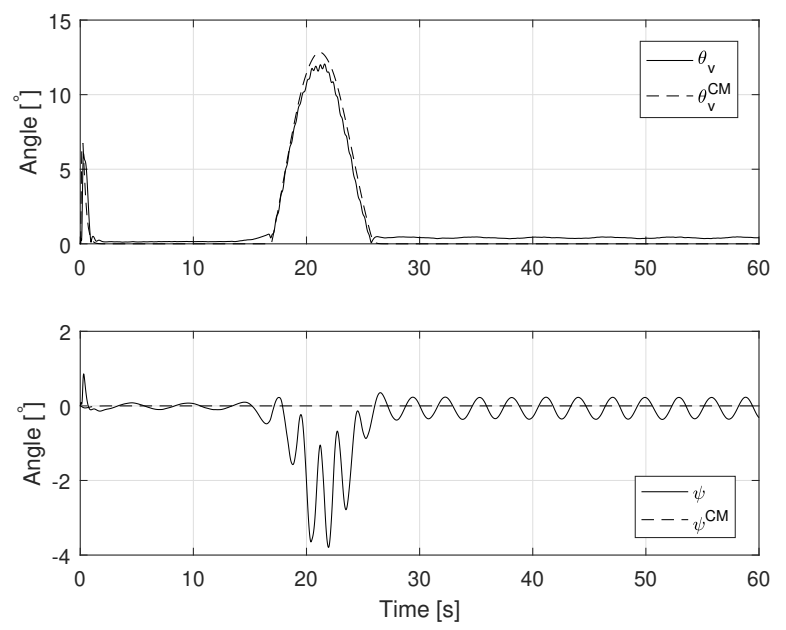

Fig. 5. Attitude tracking - Inclination (top) and yaw angle (bottom) (solid lines: multi-body model - dashed lines: control model).

Lemma 3: Given $F_{a}$ defined in (38) and the closed set $T \mathrm{SO}(3)$, we have the following:

$F_{a}\left(x_{a}\right) \cap T_{T \mathrm{SO}(3)}\left(x_{a}\right)=$ 


$$
\bigcup_{\substack{R_{p} \in \mathrm{SO}_{(3)} \\
\left\|\omega_{p}\right\| \leq \omega_{M}}}\left[\begin{array}{c}
R_{p} R_{e} R_{p}^{T} \hat{e}_{\omega} \\
-J^{-1}\left(R_{p}^{T} e_{R}+K_{\omega} e_{\omega}+\hat{e}_{\omega} J e_{\omega}+\hat{e}_{\omega} J \omega_{p}\right)
\end{array}\right] .
$$

Proof of Lemma 3. For a smooth manifold, the tangent cone is equivalent to the tangent space, namely $T_{T \mathrm{SO}(3)}\left(x_{a}\right)=$ $T_{x_{a}} T \mathrm{SO}(3)$. Since $T \mathrm{SO}(3)$ is a trivial bundle, i.e., $T \mathrm{SO}(3)=$ $\mathrm{SO}(3) \times \mathbb{R}^{3}$, then we can write $T_{\left(R_{e}, e_{\omega}\right)} T \mathrm{SO}(3) \simeq T_{R_{e}} \mathrm{SO}(3) \times$ $\mathbb{R}^{3}$. Hence, equality (62) follows from the observation that $R_{e} R_{p} \hat{e}_{\omega} R_{p}^{T} \in T_{R_{e}} \mathrm{SO}(3) \longleftrightarrow R_{p} \in \mathrm{SO}(3) . \quad \square$ To prove asymptotic stability, we employ the following invariance principle, which is a corollary of [20, Thm 1].

Proposition 3: Consider $\dot{x} \in F(x), x \in \mathcal{C}$, where $\mathcal{C}$ is a closed set, $F$ is a set-valued mapping outer semicontinuous and locally bounded relative to $\mathcal{C}, F(x)$ is nonempty and convex $\forall x \in \mathcal{C}$. Given a compact set $\mathcal{A}$, if there exists a continuously differentiable function $V$, positive definite and radially unbounded around $\mathcal{A}$ relative to $\mathcal{C}$ and such that

$$
\dot{V}(x)=\max _{f \in F(x) \cap T_{\mathcal{C}}(x)}\langle\nabla V(x), f\rangle \leq 0 \quad \forall x \in \mathcal{C} \backslash \mathcal{A},
$$

then $\mathcal{A}$ is globally stable, namely Lyapunov and Lagrange stable. Furthermore,

1) if there exists an open neighborhood $\mathcal{U} \supset \mathcal{A}$ from which no complete solution $\gamma$ exists, satisfying $V(\gamma(t)))=V(\gamma(0)) \neq$ 0 , then $\mathcal{A}$ is asymptotically stable.

2) Any such neighborhood $\mathcal{U}$ of the form

$$
\mathcal{U}:=\{x \in \mathcal{C}: V(x)<\ell\}, \ell>0,
$$

is contained in the basin of attraction of $\mathcal{A}$.

Proof of Theorem 1. Since all solutions to (28), (29) are also solution to (36), we prove Theorem 1 by applying Proposition 3 to (36) with $\mathcal{A}=\left\{I_{3}, 0\right\}=:\left\{x_{a}^{*}\right\}$, with the Lyapunov candidate $V_{R}$ in (32) which is clearly positive definite and radially unbounded. In particular, for $V=V_{R}$, we first prove (63) and then we prove item 2) of Proposition 3 with $\mathcal{U}=S_{a}$, according to (31) and (64). Verification of (63). From Lemma 3, we may evaluate $\dot{V}_{R}$ in (63) with $F=F_{a}$ and $F_{a}\left(x_{a}\right) \cap T_{T \mathrm{SO}(3)}\left(x_{a}\right)$ given in (38), as follows (where we use $e_{\omega}^{T}\left(\hat{e}_{\omega} a\right)=0$ and $\dot{\Psi}_{K_{R}}\left(R_{e}\right)=-\frac{1}{2} \operatorname{tr}\left(K_{R} R_{e}\right)=$ $\left.e_{R}^{T} R_{p} e_{\omega}\right)$

$$
\dot{V}_{R}\left(x_{a}\right)=-e_{\omega}^{T} K_{\omega} e_{\omega} \leq 0, \quad \forall x_{a} \in T \mathrm{SO}(3)
$$

Verification of item 2) of Proposition 3. According to (64) and (31), select $\mathcal{U}=\left\{x_{a} \in T \mathrm{SO}(3): V_{R}\left(x_{a}\right)<\ell\right\}$ for some $\ell>0$. Thanks to the properties of the potential function $\Psi_{K_{R}}$, the sublevel set of the form $\left\{x_{a} \in T \operatorname{SO}(3): V_{R}\left(x_{a}\right)<\ell\right\}$, where $\ell \leq \ell_{R}$, with $\ell_{R}$ defined in (30), contains only the desired equilibrium point. Indeed, $V_{R}\left(x_{a}\right)<\ell_{R}$ implies $\Psi_{K_{R}}\left(R_{e}\right)<\ell_{R}$ for any $x_{a} \in T \mathrm{SO}(3)$ and only the desired equilibrium point is contained in this sublevel set. Furthermore, since this set is forward invariant from (65) and the viability condition of [6, Pag. 124]) is satisfied, maximal solutions starting in $\mathcal{U}$ are complete. We refer now to a solution $t \mapsto \gamma(t)$ starting in $\mathcal{U}$ for which $V_{R}(\gamma(0))=a \neq 0$. Then, if we consider $\gamma(0) \in\left\{x_{a} \in \mathcal{U}: e_{\omega} \neq 0\right\}$, the function $V_{R}(\gamma(t))$ has to decrease in time by continuity, which implies $\dot{V}_{R}(\gamma(t))<0$. Instead, if $\gamma(0) \in \mathcal{G}:=\left\{x_{a} \in \mathcal{U}: e_{\omega}=0\right\} \backslash\left\{x_{a}^{*}\right\}$, then, according to the closed loop dynamics

$$
\left.F_{a}\left(x_{a}\right)\right|_{\mathcal{G}}=\bigcup_{\substack{R_{p} \in \overline{c o}(\mathrm{SO}(3)) \\
\left\|\omega_{p}\right\| \leq \omega_{M}}}\left[\begin{array}{c}
0 \\
-J^{-1} R_{p}^{T} e_{R}
\end{array}\right]
$$

$\gamma(t)$ will exit the set $\mathcal{G}$ for some small $t>t_{0}$, since $e_{R} \neq$ $0 \forall x_{a} \in \mathcal{U} \backslash\left\{x_{a}^{*}\right\}$. As a consequence, $V_{R}(\gamma(t))$ is forced to decrease again. We can conclude that there is no complete solution that keeps $V_{R}(\gamma(t))$ constant and different from zero. Therefore, from Proposition 3 the proof is complete.

\section{CONCLUSIONS}

In this paper we proposed a priority-oriented robust control paradigm for a general class of underactuated UAVs, in which position tracking is the primary objective. To this end, we exploited a dynamic attitude planner in charge of providing a modified attitude reference, which guarantees that the control force, required to track the desired position, can always be delivered. A numerical simulation has been performed by considering a hexarotor with tilted propellers to verify the robustness of the control law against unmodeled effects such as unknown mass distribution, aerodynamic drag and disturbance torques. Future work will be oriented to improve the position tracking performance by considering highperformance feedback stabilizers [4] which require a non-trivial extension of the robust design proposed here.

\section{REFERENCES}

[1] F. Bullo and R. M. Murray, "Tracking for fully actuated mechanical systems: a geometric framework," Automatica, vol. 35 , no. 1, pp. $17-$ 34, Jan 1999.

[2] Z. Cai, M. S. de Queiroz, and D. M. Dawson, "A sufficiently smooth projection operator,' IEEE Transactions on Automatic Control, vol. 51, no. 1, pp. 135-139, Jan 2006.

[3] B. Crowther, A. Lanzon, M. Maya-Gonzalez, and D. Langkamp, "Kinematic analysis and control design for a nonplanar multirotor vehicle," Journal of Guidance, Control, and Dynamics, vol. 34, no. 4, pp. 1157-1171, 2011.

[4] F. Forni, S. Galeani, and L. Zaccarian, "A family of global stabilizers for quasi-optimal control of planar linear saturated systems," IEEE Transactions on Automatic Control, vol. 55, no. 5, pp. 1175-1180, May 2010.

[5] A. Franchi, R. Carli, D. Bicego, and M. Ryll, "Full-pose tracking control for aerial robotic systems with laterally bounded input force," IEEE Transactions on Robotics, vol. PP, no. 99, pp. 1-8, 2018.

[6] R. Goebel, R. G. Sanfelice, and A. R. Teel, Hybrid Dynamical Systems. University Press Group Ltd, 2012.

[7] T. Hamel, R. Mahony, R. Lozano, and J. Ostrowski, "Dynamic modelling and configuration stabilization for an X4-Flyer." IFAC Proceedings Volumes, vol. 35, no. 1, pp. 217 - 222, 2002, 15th IFAC World Congress.

[8] M. Hua, T. Hamel, P. Morin, and C. Samson, "Control of VTOL vehicles with thrust-tilting augmentation," Automatica, vol. 52, no. 2 , pp. 1-7, feb 2015.

[9] D. Invernizzi, M. Giurato, P. Gattazzo, and M. Lovera, "Full pose tracking for a tilt-arm quadrotor UAV," in Proc. IEEE Conf. Control Technology and Applications (CCTA), Aug. 2018, pp. 159-164.

[10] D. Invernizzi, M. Lovera, and L. Zaccarian, "Dynamic attitude planning for trajectory tracking in underactuated UAVs," arXiv:1810.04494, 2018.

[11] —, "Geometric trajectory tracking with attitude planner for vectored-thrust VTOL UAVs," in American Control Conference, Milwaukee (WI), USA, Jul. 2018.

[12] D. Invernizzi and M. Lovera, "Trajectory tracking control of thrustvectoring UAVs," Automatica, vol. 95, pp. 180 - 186, 2018.

[13] T. Lee, M. Leok, and H. McClamroch, "Geometric tracking control of a quadrotor UAV on SE(3)," in IEEE Conference on Decision and Control, Atlanta, USA, 2010.

[14] M. Maggiore, M. Sassano, and L. Zaccarian, "Reduction theorems for hybrid dynamical systems," IEEE Transaction on Automatic Control, 2019.

[15] C. G. Mayhew, R. G. Sanfelice, and A. R. Teel, "Quaternion-based hybrid control for robust global attitude tracking," IEEE Transactions on Automatic Control, vol. 56, no. 11, pp. 2555-2566, Nov 2011.

[16] G. Michieletto, A. Cenedese, L. Zaccarian, and A. Franchi, "Nonlinear control of multi-rotor aerial vehicles based on the zero-moment direction," in IFAC 20 th World Congress, Toulouse, France, 2017.

[17] R. Naldi, M. Furci, R. G. Sanfelice, and L. Marconi, "Robust global trajectory tracking for underactuated VTOL aerial vehicles using inner-outer loop control paradigms," IEEE Transactions on Automatic Control, vol. 62, no. 1, pp. 97-112, 2017.

[18] A. Roza and M. Maggiore, "A class of position controllers for underactuated VTOL vehicles," IEEE Transactions on Automatic Control, vol. 59, no. 9, pp. 2580-2585, 2014.

[19] M. Ryll, H. H. Bülthoff, and P. Robuffo Giordano, "A novel overactuated quadrotor unmanned aerial vehicle: Modeling, control, and experimental validation," IEEE Transactions on Control Systems Technology, vol. 23, no. 2, pp. 540-556, Mar. 2015.

[20] A. Seuret, C. Prieur, S. Tarbouriech, A. R. Teel, and L. Zaccarian, "A nonsmooth hybrid invariance principle applied to robust eventtriggered design," IEEE Transactions on Automatic Control, p. 1, 2018 
[21] S. Zhao, W. Dong, and J. A. Farrell, "Quaternion-based trajectory, tracking control of VTOL-uavs using command filtered backstepping," in Proc. American Control Conf, Jun. 2013, pp. 1018-1023. 